\title{
INFLUÊNCIA DO TREINAMENTO AERÓBIO NOS MECANISMOS FISIOPATOLÓGICOS DA HIPERTENSÃO ARTERIAL SISTÊMICA
}

\author{
DR. FRANCISCO LUCIANO PONTES JÚNIOR \\ Doutor em Ciências/Reabilitação pela Universidade Federal de São Paulo (UNIFESP) e \\ Professor do Curso de Gerontologia da Escola de Artes, Ciências e Humanidades da \\ Universidade de São Paulo -EACH/USP (São Paulo - Brasil) \\ e-mail: lucianopontes@usp.br \\ DR. JONATO PRESTES \\ Doutor em Ciências Fisiológicas pela Universidade Federal de São Carlos (UFSCar) e \\ Professor do Programa de Mestrado e Doutorado em Educação Física da \\ Universidade Católica de Brasília (UCB) (Distrito Federal - Brasil) \\ e-mail: jonatop@gmail.com
}

\section{MS. RICHARD DIEGO LEITE}

Mestre em Ciências Fisiológicas pela Universidade Federal de São Carlos (UFSCar) e Doutorando do Programa de Biociências da Universidade do Estado do Rio de Janeiro (UERJ). Departamento de Ciências Fisiológicas Laboratório de Pesquisas Clínicas e Experimentais em Biologia Vascular (BioVasc),

Centro Biomédico (Rio de Janeiro - Brasil) e-mail: rdleite@gmail.com

\section{GRAD. DANIEL RODRIGUEZ}

Graduado em Educação Física pelo Centro Universitário das Faculdades Metropolitanas Unidas (FMU). Escola de Artes, Ciências e Humanidades da Universidade de São Paulo-EACH/USP.

Curso de Gerontologia (São Paulo - Brasil) e-mail:d-rodriguez@uol.com.br 


\section{RESUMO}

O objetivo da presente revisão foi discutir as principais influências do exercício aeróbio nos mecanismos fisiopatológicos da hipertensão sistêmica. A hipotensão pós-exercício (HPE) resulta de uma redução persistente na resistência vascular periférica (RVP), mediada pelo sistema nervoso autônomo e por substâncias vasodilatadoras. A diminuição da pressão arterial com o treinamento crônico ocorre pela diminuição da RVP e do débito cardíaco em repouso, por meio da redução da atividade neural simpática e do aumento da sensibilidade barorreflexa. Além disso, o exercício crônico pode promover redução da concentração de catecolaminas, melhora do perfil metabólico, afetar a atividade funcional do endotélio vascular e promover mudanças positivas na composição corporal. Desse modo, a inclusão do exercício físico aeróbio é fortemente recomendada como estratégia não farmacológica para o tratamento da hipertensão, não apenas pelo efeito benéfico na pressão arterial, bem como na redução de fatores de risco cardiovasculares.

PALAVRAS-CHAVE: Exercício; pressão arterial; hipertensão e hipotensão.

\section{INTRODUÇÃO}

A hipertensão é uma doença que afeta aproximadamente dois a três adultos em cada 10 e contribui de forma expressiva para um grande número de mortes decorrentes de infarto do miocárdio, acidente vascular encefálico e doença renal crônica (ACSM, 2004). A relação entre pressão arterial elevada e risco de eventos cardiovasculares é contínua, consistente e independente de outros fatores de risco (CHOBANIAN et al. , 2003).

O treinamento físico aeróbio reduz a pressão arterial em 75\% dos indivíduos com hipertensão, sendo um componente chave na terapia não farmacológica para sua prevenção e tratamento. (GOODWIN; HEADLEY; PESCATELLO, 2009). O exercício físico no tratamento tem como objetivo reduzir a morbi-mortalidade, o que pode ser obtido com modificações dos níveis de atividade física e hábitos de vida em associação com o tratamento farmacológico (MANFREDINI et al. , 2009). Além disso, outras modificações como, a redução do percentual de gordura corporal, da ingestão de sódio e moderação no consumo de álcool (CHOBANIAN et al. , 2003) são outras condutas importantes para o controle da pressão arterial.

Adicionalmente, o exercício físico tem demonstrado forte influência na redução de fatores de risco para doenças cardiovasculares, assim como sobrepeso e obesidade, distúrbios do metabolismo lipídico, resistência à insulina e intolerância a glicose (PONTES et al. , 2008). Apesar da influência do exercício físico nos fatores de risco salientados acima, ainda é necessária uma visão integrada dos efeitos agudos e crônicos do exercício aeróbio sobre os mecanismos fisiopatológicos da hipertensão. Desse modo, foram discutidos os principais mecanismos fisiopatológicos envolvidos 
na hipertensão, efeitos agudos e crônicos do exercício aeróbio sobre a pressão arterial e os possíveis mecanismos pelos quais o exercício aeróbio promove reduções pressóricas.

\section{METODOLOGIA}

$\bigcirc$ presente estudo incluiu pesquisas bibliográficas com base descritiva e exploratória relacionada a exercício físico aeróbio e hipertensão. $\bigcirc$ primeiro passo para a construção desta revisão foi à identificação de estudos pertinentes em busca on-line em base de dados científicas. Foram usados os periódicos do Portal Capes, Pubmed, Scielo, Highwire, Isis of Knowledge e Lilacs. Uma análise semiquantitativa do material encontrado na literatura for realizada baseado nos principais achados sobre $\mathrm{o}$ assunto.

\section{EFEITO AGUDO DO EXERCíCIO FÍSICO AERÓBIO NA HIPERTENSÃO}

Estudos têm relatado redução da pressão arterial em indivíduos normotensos e hipertensos após uma única sessão de exercício físico (BROWLEY et al., 2003; KULICS; COLLINS; DICARLO, 1999; LEGRAMANTE et al., 2002; RONDON; BRUM, 2003; SENITKO; CHARKOUDIAN; HALLIWIL, 2002). Embora a hipotensão pós-exercício (HPE) seja detectada em normotensos, nesses indivíduos a resposta pressórica parece ser menos consistente e de menor magnitude do que em indivíduos hipertensos (MACDONALD, 2002). Em indivíduos normotensos a queda da pressão arterial sistólica e diastólica é aproximadamente de 8 a $10 \mathrm{mmHg}$ e 3 a $5 \mathrm{mmHg}$, respectivamente. Já em indivíduos hipertensos, essa diminuição é de aproximadamente 18 a $20 \mathrm{mmHg}$ para pressão arterial sistólica e de 7 a $9 \mathrm{mmHg}$ para a pressão arterial diastólica (KENNEY; SEALS, 1993).

A HPE parece ser mantida na maior parte das 24 horas subsequentes ao exercício físico, com redução de maior magnitude em indivíduos hipertensos (GOODWIN; HEADLEY; PESCATELLO, 2009).

\section{MECANISMOS ENVOLVIDOS NA HIPOTENSÃO PÓS-EXERCÍCIO (HPE)}

Alguns autores postulam que o mecanismo da HPE esteja ligado à redução da RVP (LEGRAMANTE et al., 2002), redução da atividade simpática (BROWLEY et al., 2003; KULICS; COLLINS; DICARLO, 1999) e redução do débito cardíaco associado ao menor volume sistólico (NEGRÃO; RONDON, 200 I; RONDON; BRUM, 2003). Segundo Halliwill (200 I) a HPE resulta da redução na RVP, mediada pelo sistema nervoso autônomo e substâncias vasodilatadoras. De fato, a redução 
na atividade nervosa simpática é uma característica evidente na HPE (KULICS; COLLINS; DICARLO, 1999) e tem sido proposta como mediadora da diminuição da pressão arterial após o exercício (CHANDLER; DICARLO, 1997). Em indivíduos hipertensos, as reduções na pressão arterial e na RVP após uma sessão de exercício aeróbio ocorrem sem aumentos correspondentes na frequência cardíaca, na atividade nervosa simpática e nas concentrações plasmáticas de noradrenalina (CHANDLER; RODENBAUGH; DICARLO, 1998).

De acordo com Dicarlo e Bishop (200I) em condições de repouso, a atividade simpática no músculo é fortemente regulada pelos pressorreceptores arteriais e cardiopulmonares. No início do exercício aeróbio, a frequência cardíaca e a atividade do sistema nervoso simpático aumentam abruptamente. Nessa situação, ocorre o reajuste dos pressorreceptores em faixas de funcionamento mais elevadas e ocorre o aumento da atividade simpática. Após o exercício aeróbio, ocorre o reajuste para pressões mais baixas de tal forma que a ativação simpática vindo do sistema nervoso central diminui, ficando menor que nos níveis pré-exercício, o que induz a diminuição da RVP e da pressão arterial.

De acordo com Halliwill (200 I); Chen e Bonham (20 l0), após o exercício, a queda da pressão arterial pode ocorrer por componentes neurais e vasculares, vasodilatação sustentada, que estão associados à redução do efeito vasoconstritor das catecolaminas. $\bigcirc$ componente neural refere-se à diminuição da atividade simpática no músculo (que envolve o reajuste dos pressorreceptores). . O componente vascular está relacionado à atenuação da sensibilidade ao estímulo simpático, ou seja, menor resposta vascular à ativação dos receptores alfa-adrenérgicos, bem como à influência potencial de substâncias vasodilatadoras.

Segundo Rao, Collins e DiCarlo (2002) após o exercício ocorre redução significativa da capacidade da vasculatura para responder à alterações da atividade simpática ou ao aumento das catecolaminas. No que se refere à influência de substâncias vasodilatadoras, durante o exercício o sangue é redistribuído aos músculos esqueléticos, provocando vasodilatação que não está restrita aos locais dos músculos esqueléticos ativos, envolvendo também regiões inativas (HALLIWILL, 200 I). O maior fluxo sanguíneo, com consequente distensão dos vasos, parece induzir o aumento do estresse de cisalhamento, mecanismo ligado à síntese e liberação do óxido nítrico (GOTO et al., 2003). De fato, em estudos realizados com humanos, o exercício aumenta a produção de óxido nítrico (JUNGERTEN et al., 1997) e atenua a resposta vasoconstritora associada à estimulação dos receptores alfa-adrenérgicos. Além do óxido nítrico, outras substâncias vasodilatadoras também podem modificar a resposta vasoconstritora alfa-adrenérgica, tais como as prostaglandinas, adenosina, ATP, potássio, lactato, bradicina e a vasopressina (HALLIWILL; 2001 ; PONTES et al., 2008). 
Segundo Negrão e Rondon (200 I) a HPE tem importância clínica, uma vez que os níveis da pressão arterial 24 horas após uma sessão de exercício físico permanecem abaixo dos níveis pressóricos de um dia sem exercício físico. Portanto, o exercício agudo pode representar uma abordagem terapêutica segura para a redução da pressão arterial em indivíduos hipertensos (KULICS; COLLINS; DICARLO, 1999).

\section{EFEITO DO FÍSICO AERÓBIO CRÔNICO NA PRESSÃO ARTERIAL}

O treinamento físico exerce um papel positivo na prevenção da hipertensão e na redução da pressão arterial em indivíduos com níveis pressóricos elevados (ACSM, 2004; FAGARD, I999; FAGARD, 200 I; ISHIKAWA-TAKATA; OHTA; TANAKA, 2003; MIYAl et al., 2002, WHELTON et al., 2002; MANFREDINI et al., 2009). Dentre os mecanismos pelos quais o exercício aeróbio pode reduzir a pressão arterial de forma crônica, destacam-se a redução da RVP e do débito cardíaco em repouso por meio da redução da atividade simpática e o aumento da sensibilidade dos pressorreceptores (BRUM et al., 2000; STEWART, 2003).

O exercício aeróbio parece favorecer a redução dos níveis plasmáticos das catecolaminas, interfere na função endotelial, melhora tanto o estado hiperinsulinêmico (GOTO et al. , 2003; TADDEl et al., 2000), bem como o perfil lipoprotéico, contribuindo para a perda de massa corporal (GRUNDY et al., 2004; PONTES et al., 2008)

\section{TREINAMENTO AERÓBIO E SISTEMA NERVOSO SIMPÁTICO}

Um dos principais efeitos do treinamento aeróbio é sobre o tônus simpático (KRIEGER; DASILVA; NEGRÃO, 200I). O exercício crônico reduz a atividade nervosa simpática em repouso, sendo essa redução mais pronunciada em indivíduos hipertensos do que em normotensos (O'SULLIVAN; BELL, 2000). O mecanismo que explica esse efeito do treinamento é a melhora da sensibilidade dos pressorreceptores que está prejudicada na hipertensão (ACSM, 2004; O'SULIVAN; BELL, 2000; RAO; COLLINS; DICARLO, 2002).

Brum et al., (2000) constataram que o treinamento melhorou a sensibilidade dos pressorreceptores em ratos normotensos espontaneamente hipertensos. Nesse caso, um dos mecanismos propostos para explicar esse processo foi o aumento da complacência arterial, o que pode ter melhorado a sensibilidade barorreflexa. Outra hipótese usada para explicar essa melhora são as alterações endoteliais que ocorrem com o treinamento. Tanto a magnitude quanto a frequência do estresse de cisalhamento nas células endoteliais durante o exercício aumenta a liberação de 
fatores endoteliais e/ou à sensibilidade das células endoteliais, melhorando a atividade dos pressorreceptores. A melhora da sensibilidade barorreflexa e a redução da atividade simpática também foram observadas em indivíduos hipertensos de meia idade após 12 semanas de exercício aeróbio moderado (HUA et al., 2009).

Em homens sedentários saudáveis, o barorreflexo cardiovagal está mais fortemente relacionado à complacência arterial das carótidas, sendo que, o efeito positivo do exercício está associado a melhoras na complacência dessas artérias (MANAHAN et al., 200 la). Desse modo, uma das hipóteses levantadas para explicar a melhora seria a tensão de ruptura nas fibras de colágeno, provocado por pressão mecânica distensora durante o exercício. Adicionalmente, a complacência pode ser alterada por curto período de tempo pela modulação do tônus simpático-adrenérgico das células musculares lisas da parede arterial. Portanto, é possível que o treinamento aumente a complacência pela redução do controle crônico exercido pelo tônus simpático-adrenérgico, tanto de forma direta, ou pelo aumento do efeito inibidor do óxido nítrico sobre a atividade simpática. (MANAHAN et al., 200 la)

Concomitante a redução da atividade simpática induzida pelo exercício crônico, reduções da noradrenalina plasmática após o treinamento tem sido observadas (MEREDITH et al., 199I). Brown et al., (2002) verificaram reduções da taxa de liberação de noradrenalina após treinamento aeróbio de seis meses em idosos hipertensos. A supressão da atividade do sistema simpático pode contribuir para a redução da pressão arterial média e é responsável em parte pela heterogeneidade na resposta da pressão arterial ao treinamento aeróbio em idosos (BROWN et al., 2002).

TREINAMENTO AERÓBIO E FUNÇÃO ENDOTELIAL - EFEITOS NA RESPONSIVIDADE E ESTRUTURA VASCULAR

Uma importante adaptação ao treinamento aeróbio associada à redução da RVP é a melhora da função endotelial (GOTO et al., 2003; TADDEl et al. , 2000). O treinamento induz muitas adaptações musculares como a angiogênese nos músculos ativos, causando aumento da capilaridade (PORTER et al., 2002; PRIOR et al., 2003) e consequente incremento do consumo máximo de oxigênio $\left(\mathrm{VO}_{2} \max \right)$ (RHEAUMÉ et al., 2003).

A melhora da capacidade oxidativa e da massa de células endoteliais induzida pelo treinamento é acompanhada pelo aumento na atividade de duas enzimas endoteliais, a óxido-nítrico sintase e lipoproteína-lipase. Há também evidências que a atividade da óxido nítrico-sintase endotelial nas artérias que suprem o músculo esquelético fica aumentada em resposta ao estresse de cisalhamento resultante do maior fluxo sanguíneo. Este processo causa melhora na regulação da resposta vasodilatadora (dependente do fluxo sanguíneo) nos grandes vasos (GOTO et al., 
2003; JUNGERTEN et al., 2003; MAIORANA et al., 2003). O treinamento aeróbio induz ao remodelamento vascular e ao aumento na capilarização sem a hipertrofia das fibras musculares. Além disso, o exercício induz a arteriogênese, que é a ampliação dos vasos condutores já existentes, aumentando a capacidade de fluxo para o músculo (PRIOR et al., 2003).

Nesse sentido, é relevante ressaltar que o óxido nítrico está implicado nos dois processos supracitados e medeia o remodelamento vascular em resposta ao treinamento (DINENNO et al., 200 I; LLOYD; YANG; TERJUNG, 200 I). Higashi et al., ( 1999) estudaram o efeito crônico do exercício aeróbio sobre a função endotelial em indivíduos com hipertensão primária. Os autores concluíram que o exercício, além de reduzir a pressão arterial, pode melhorar a liberação de óxido nítrico induzida pela acetilcolina. Outro importante efeito do exercício regular parece ser a redução dos níveis de endotelina e alteração da resposta vascular a esse vasoconstritor (MAEDA et al., 200।; MAEDA et al., 2003).

O treinamento aeróbio também pode prevenir a disfunção endotelial associada ao envelhecimento (DESOUZA et al., 2000; TADDEl et al., 2000). Um dos mecanismos envolvidos no processo do aumento da vasodilatação dependente do endotélio é o aumento da produção e liberação de óxido nítrico. Outros mecanismos envolvidos na melhora da função vasodilatadora endotelial é o aumento da liberação de prostaglandinas, redução de radicais livres (mediada pela degradação do óxido nítrico) e redução do tônus vasoconstritor simpático (DESOUZA et al., 2000)

\section{TREINAMENTO AERÓBIO E OS SISTEMAS CALICREÍNA-CININAS E RENINA- ANGIOTENSINA}

O sistema renina-angiotensina e calicreína-cininas interagem em vários níveis. Aumento nas concentrações séricas da enzima conversora da angiotensina (ECA) pode resultar em maior síntese de angiotensina II e desativação das cininas. Enquanto a angiotensina II age estimulando a reabsorção de quantidades elevadas de sódio, aumentando a pressão arterial e causando danos nos órgãos-alvo, as cininas têm um efeito oposto, regulando o balanço de água e sódio e a pressão arterial (TAN et al., 2004; SCHMAIER, 2003; IRIGOVEN et al., 2003; OLIVEIRA et al., 2003; BADER, 200 I; CAMPBELL, 2000; DENDORFER et al., 1999).

O sistema calicreína-cininas também tem sido descrito como tendo um papel relevante na etiologia da hipertensão e na patogênese dos danos cardíacos e renais a ela associados (SHARMA \& SHARMA, 2002; DENDORFER et al., 200 I; BADER, 200 I). As cininas são proteínas, que agem através de dois tipos de receptores, o tipo I (B- I) e o tipo 2 (B-2). Os receptores B-2 normalmente predominam, enquanto os receptores B- I são ativados por danos nos tecidos, tais como os que ocorrem após uma isquemia do miocárdio ou inflamação. Uma complexa variedade de cininas age através desses 
receptores. No homem, a calicreína plasmática forma a bradicinina, a partir do cininogênio, enquanto a calicreína nos tecidos forma a calidina (CAMPBELL, 2000).

Os peptídios cininas têm ação importante nos vasos sanguíneos, no coração e nos rins. Um efeito hemodinâmico relevante das cininas, "in vivo", é a vasodilatação produzida pela estimulação dos receptores endoteliais B-2, nas artérias e arteríolas, com subsequente liberação de óxido nítrico e prostaglandinas (SCHMAIER, 2003; DUNCAN et al., 2000). As cininas estão implicadas em vários processos fisiológicos e patológicos, podendo exercer uma influência significativa na regulação da pressão arterial, na homeostase do sódio e no tônus vascular, devido à sua potente atividade vasodilatadora, natriurética e moduladora do crescimento vascular. (SHARMA, 2003; SHARMA \& SHARMA, 2002; DENDORFER et al., 200 I; DENDORFER et al., 1999).

Miura, (1994) verificaram um aumento na atividade da calicreína urinária em pacientes com hipertensão primária, os voluntários realizaram treinamento aeróbio em cicloergômetro durante dez semanas (3 vezes por semana, durante 60 minutos), e este aumento ocorria nas primeiras 4 semanas após iniciado o treinamento, com significativa redução na pressão arterial. Confirmando estes resultados, SAKAl et al., ( 1998) encontraram que após 4 semanas de exercício aeróbio em bicicleta ergométrica abaixo do limiar de lactato (3 vezes por semana, durante 60 minutos), um aumento nas concentrações de calicreína urinária apenas nos pacientes hipertensos treinados, não ocorreu nenhuma diferença significativa no grupo controle sem exercício, este aumento foi de aproximadamente $184 \%$ do basal. Os autores concluíram que treinamento aeróbio moderado estimula o sistema calicreínas-cininas renal, resultando em natriurese e redução na pressão arterial em pacientes hipertensos. Pontes et. al. (2008) também observaram um aumento da calicreína e da bradicinina e queda de pressão, após a realização de 45 minutos de exercício aeróbio agudo dentro e fora da água. Além disso, a atividade da calicreína plasmática foi estudada em ratos submetidos ao exercício aeróbio, mostrando que o exercício foi capaz de induzir aumento na concentração plasmática de calicreína e concomitante aumento na captação de glicose no músculo esquelético (VETTOR et al., 1986).

Um outro efeito potencial do exercício físico regular que pode contribuir no controle da pressão arterial é a redução dos níveis de renina e angiotensina II (ACSM, 2004). Em estudo sobre os mecanismos envolvidos nas adaptações do fluxo sanguíneo com o treinamento, McAllister ( 1998) afirma que indivíduos treinados possuem menor vasoconstrição esplânica e renal durante o exercício. Segundo o autor, esse efeito está associado a reduções da ativação simpática, concentrações plasmáticas de angiotensina II e vasopressina. Outros estudos também relatam redução dos níveis de renina e angiotensina II após o treinamento em indivíduos normotensos (HESPEL et al., 1988). Entretanto, em indivíduos hipertensos os estudos ainda não são conclusivos. 
Dubbert et al. (1994) associam a atenuação da pressão arterial em indivíduos com hipertensão leve à redução da atividade da renina plásmatica. Outro autor relata que o treinamento não reduz os níveis de renina e angiotensina II de forma consistente (URATA et al., 1987). Portanto, a relação entre o treinamento e o sistema renina-angiotensina, especialmente em indivíduos hipertensos precisa ser melhor investigada, dada a sua importância na regulação do volume sanguíneo e da pressão arterial.

\section{CONSIDERAÇÕES FINAIS}

A partir das evidências científicas apresentadas nesta revisão torna-se evidente os efeitos positivos do exercício aeróbio agudo e crônico sobre a hipertensão e seus mecanismos fisiopatológicos. Os possíveis processos envolvidos no efeito antihipertensivo do exercício incluem tanto mecanismos diretos, tais como a redução da atividade simpática, o aumento da sensibilidade barorreflexa e a melhora da função endotelial, como indiretos, como a redução da obesidade e a melhora do perfil metabólico Desse modo, a inclusão do exercício físico regular é altamente recomendável como conduta não-farmacológica no tratamento da hipertensão, não apenas pelo efeito benéfico sobre a pressão arterial, bem como na redução de fatores de risco cardiovasculares. No entanto, é importante salientar que em alguns casos, somente o exercício físico aeróbio não é capaz de promover reduções significativas nos índices pressóricos, sendo necessário o uso de medicamentos. Nestes casos, a prescrição do exercício físico aeróbio regular pode contribuir positivamente para a redução da necessidade do medicamento.

\section{Influence of aerobic exercise on physiopathological mechanisms of systemic hypertension}

ABSTRACT: The aim of the present review was to discuss the main influence of aerobic exercise on the physiopathological mechanisms of systemic hypertension. Post-exercise hypotension (PEH) results from persistent reductions in peripheral vascular resistance (PVR), mediated by the autonomic nervous system and vasodilator substances. Exercise training lowers blood pressure through a decrease in total peripheral resistance and cardiac output at rest, as a result of reductions in sympathetic neural activity and increases in baroreflex sensitivity. Additionally, chronic exercise may reduce the levels of serum catecholamines, improve the metabolic profile, modulate the functional activity of the vascular endothelium, and promote positive changes on body composition. Thus, aerobic exercise is strongly recommended as a non pharmacologic strategy in the treatment of hypertension, not only for its effects on blood pressure, but also by decreasing the risk factors of cardiovascular disease.

KEYWORDS: Exercise; blood pressure; hypertension and hypotension. 


\section{Influencia del entreinamento aeróbico em los mecanismos fisiopatologia de la hipertensíon}

RESUMEN: El presente articulo tiene la intención de, a través de una revision de la literatura, verificar los efectos del ejercicio físico aeróbico a la fisiopatología de la hipertensión arterial sistémica. La hipotensión pos ejercicio (HPE) es el resultado de una reducción persistente de la resistencia vascular periférica (RVP), mediada por el sistema nervioso autónomo y sustancias vasodilatadoras. La disminución de la tensión arterial se produce con el entrenamiento crónico por la redución de la RVP y el gasto cardíaco en reposo, mediante la reducción de la actividad nerviosa simpática y aumento de la sensibilidad barorrefleja. Por otra parte, el ejercicio crónico puede disminuir la concentración de catecolaminas, mejorar el perfil metabólico, afectar a la actividad funcional del endotelio vascular y promover cambios positivos en la composición corporal. Así, se recomienda la inclusión del ejercicio aeróbico como una estrategia para el tratamiento no medicamentoso de la hipertensión, no sólo por el efecto beneficioso sobre la tensión arterial como tambien para reducir los factores de riesgo cardiovascular.

PALABRAS CLAVE: Ejercicio; presión arterial; hipertensión y hipotensión.

\section{REFERÊNCIAS}

BEEVERS, G.; LIP, G. Y. H.; O'BRIEN, E. ABC of hypertension: the pathophysiology of hypertension. BMJ, London, v. 322, n. 7291, p. 912-916, Apr. 2001.

BOIX, F. et al. Contraction-related factors affect the concentration of a kallidin-like peptide in rat muscle tissue. J Physiol, London, v. 544, n. I, p. I27- I36, Oct. 2002.

BROWN, M. D. et al. Sympathetic activity and the heterogeneous blood pressure response to exercise training in hypertensives. J Appl Physiol, Bethesda, v. 92, n. 4, p. |434-|442, Apr. 2002.

BROWNLEY, K. A. et al. Sympathoadrenergic mechanisms in reduced hemodynamic stress responses after exercise. Med Sci Sports Exerc, Madison, v. 35, n. 6, p. 978-986, Jun. 2003.

BRUM, P. C. et al. Exercise training increases baroreceptor gain sensivity in normal and hypertensive rats. Hypertension, Baltimore, v. 36, n. 6, p. 1018-1022, Dec. 2000.

CHEN, C. Y.; BONHAM, A. C. Postexercise hypotension: central mechanisms. Exerc Sport Sci Rev, Madison, v. 38, n. 3, p. 122-127, Jul. 2010.

CAMPBELL, D. J. Towards understanding the kallikrein-kinin system: insight from measurement of kinin peptides. Braz J Med Biol Res, Ribeirão Preto, v. 33, n. 6, p. 665-677, Jun. 2000.

CHANDLER, M. P.; DICARLO, S. E. Sinoartic denervation prevents postexercise reductions in arterial pressure and cardiac sympathetic tonus. Am J Physiol Heart Circ Physiol, Bethesda, v. 273, n. 6, p. 2738-2745, Dec. 1997. 
CHANDLER, M. P.; RODENBAUGH, D. W.; DICARLO, S. E. Arterial baroreflex resetting mediates postexercise reductions in arterial pressure and heart rate. Am J Physiol Heart Circ Physiol, Bethesda, v. 275, n. 5, p. 1627- 1634, Nov. 1998.

CHOBANIAN, A. V.; et al. The Seventh Report of the Joint National Committee on Prevention, Detection, Evaluation, and Treatment of High Blood Pressure: the JNC 7 report. JAMA, v. 289, n. 19, p. 2560-257I, May. 2003.

CONSOLIM-COLOMBO, F; KRIEGER, E. M. Sistema nervoso simpático e hipertensão arterial. Hipertensão, São Paulo, v. 3, n. 3, p. 86-89, jul./agost. 2000.

DAVIGNON, J.; GANZ, P. Role of endothelial dysfunction in atherosclerosis. Circulation, Dallas, v. 109, n. 23, p. 27-32, Jun. 2004.

DAVY, K.; HALL, J. E. Obesity and hypertension: two epidemics or one? Am J Physiol Regul Integr Comp Physiol, Bethesda, v. 286, n. 5, p. 803-804, May. 2004.

DENDORFER, A. et al. Pathways of bradykinin degradation in blood and plasma of normotensive and hypertensive rats. Am J Physiol Heart Circ physiol, Bethesda, v. 280, n. 5 , p. 2182- 2188, May. 2001.

DESOUZA, C. A. et al. Regular aerobic exercise prevents and restores age-related declines in endothelium-dependent vasodilation in healthy men. Circulation, Dallas, v. 102, n. 12, p. |35 | - 357, Sep. 2000.

DIBONA, G. F. Peripheral and central interactions between the renin-angiotensin system and the renal sympathetic nerves in control of renal function. Ann NY Acad Sci, New York, v. 940, n. I, p. 395-406, Jun. 2001.

DICARLO, S. E.; BISHOP, V. S. Central barorreflex resetting as a means of increasing and decreasing sympathetic outflow and arterial pressure. Ann N Y Acad Sci, New York, v. 940, n. I, p. 324-337, Jun. 2001.

DIETZE, G. J. et al. Potencial role of bradykinin in forearm muscle metabolism in humans. Diabetes, New York, v. 45, n. I, p. I I0- I |4, Jan. 1996.

DINENNO, F. A. et al. Regular endurance exercise induces expansive arterial remodeling in the trained limbs of healthy men. J Physiol, London, v. 534, n. I, p. 287-295, Jul. 200 I.

DUBBERT, P. M. et al. Endurance exercise in mild hypertension: effects on blood pressure and associated metabolic and quality of life variables. J Hum Hypertens, London, v. 8, n. 4, p. 265-272, Apr. 1994.

FAGARD, R. H. Physical activity in the prevention and treatment of hypertension in the obese. Med Sci Sports Exerc, Baltimore, v. 3 I, n. I I, p. 624-630, Nov. 1999. 
FAGARD, R. H. Exercise characteristics and the blood pressure response to dynamical physical training. Med Sci Sports Exerc, Baltimore, v. 33, n. 6, p. 484-492, Jun. 2001.

GONÇALVES, A. R. R.; ZATZ, R.; HEIMANN, J. C. O papel do rim no controle da pressão arterial. Hipertensão, São Paulo, v. 3, n. I, p. 6-13, jan./marc. 2000.

GOODYEAR, L. J.; KAHN, B. B. Exercice, glucose transport and insulin sensivity. Annu Rev Med, Pelo Alto, v. 49, n. I, p. 235-26I, Feb. 1998.

GOODWIN, K. A.; HEADLEY, S. A. E.; PESCATELLO, L. S. Physical Activity: Exercise prescription for the prevention and management of hypertension. Am J Lifestyle Med, Thousand Oaks, v. 3, n. 6, p. 446 - 449, Nov. 2009.

GOTO, C. et al. Different intensities of exercise on effect of endothelium-dependent vasodilation in humans. Circulation, Dallas, v. I08, n. 5, p. 530-535, Jul. 2003.

GRASSI, G. et al. Adrenergic and reflex abnormalities in obesity-related hypertension. Hypertension, Baltimore, v. 36, n. 4, p. 538-542, Oct. 2000.

GRUNDY, S. M. et al. Clinical management of metabolic syndrome: report of the American Heart Association/ National Heart, Lung, and Blood Institute/American Diabetes Association conference on scientific issues related to management. Circulation, Dallas, v. I09, n. 4, p. 55।-556, Feb. 2004.

HAGBERG, J. M.; MONTAIN, S. J.; MARTIN, W. H. $3^{\text {rd. }}$. Blood pressure and hemodynamic responses after exercise in older hypertensives. J Appl Physiol, Bethesda, v. 63, n. I, p. 270276, Jul. 1987.

HALLIWILL, J. R. Mechanisms and clinical implications of post-exercise hypotension in humans. Exerc Sport Sci Rev, Baltimore, v. 29, n. 2, p. 65-70, Apr. 2001.

Heart and Stroke Foundation of Canada. The Growing Burden of Heart Disease and Stroke. Ottawa: Heart \& Stroke Foundation of Canada, May. 2003.

HESPEL, P. et al. Effects of physical endurance training on the plasma renin-angiotensinaldosterone system in normal man. J Endocrinol, Bristol, v. I 16, n. 3, p. 443-449, Mar. 1988.

HIGASHI, Y. et al. Regular aerobic exercise augments endothelium-dependent vascular relaxation in normotensive as well as hypertensive subjects: role of endothelium-derived nitric oxide. Circulation, Dallas, v. 100, n. II, p. I194-1202, Sep. 1999.

HUA, L. P. T.; et al. Effects of Low-Intensity Exercise Conditioning on Blood Pressure, Heart Rate, and Autonomic Modulation of Heart Rate in Men and Women with Hypertension. Biol Res Nurs, Thousand Oaks, v. I I, n. 2, p. 129 - I43, Oct. 2009. 
ISHIKAWA-TAKATA, K.; OHTA, T.; TANAKA, H. How much exercise is required to reduce blood pressure in essential hypertensives: a dose-response study. Am J Hypertens, New York, v. 16, n. 8, p. 629-633, Aug. 2003.

IZZO, J. L. JR.; TAYLOR, A. A. The sympathetic nervous system and baroreflexes in hypertension and hypotension. Curr Hypertens Rep, Philadelphia, v. I, n. 3, p. 254-263, Jun. 1999.

JONES, P. P. et al. Baroreflex buffering is reduced with age in health men. Circulation, Dallas, v. 107, n. 13, p. 1770-1774, Apr. 2003.

JUNGERSTEN, L. et al. Both physical fitness and acute exercise regulate nitric oxide formation in healthy humans. J Appl Physiol, Bethesda, v. 82, n. 3, p. 760-764, Mar. 1997.

KENNEY, M. J.; SEALS, D. R. Hypotension, key features, mechanisms and clinical significance. Hypertension, Baltimore, v. 22, n. 5, p. 653-664, Nov. 1993.

KISHI, K. et al. Bradykinin directly triggers GLUT4 translocation via an insulin-independent pathway. Diabetes, Alexandria, v. 47, n. 4, p. 550-558, Apr. 1998.

KOHLMAN, O, JR. et al. Role of bradykinin in insulin sensivity and blood pressure regulation during hyperinsulinemia. Hypertension, Dallas, v. 25, n. 5, p. 1003-1007, May. 1995.

KRIEGER, E. M.; DA SILVA, G. J.; NEGRÃO, C. E. Effects of exercise training on baroreflex control of the cardiovascular system. Ann N Y Acad Sci, New York, v. 940, n. I, p. 338-347, Jun. 2001 .

KULICS, J. M.; COLLINS, H. L.; DICARLO, S. E. Postexercise hypotension is mediated by reductions in sympathetic nerve activity. Am J Physiol., Bethesda, v. 276, n. I, p. 27-32, Jan. 1999.

LANFRANCHI, P. A.; SOMERS, V. K. Arterial baroreflex function and cardiovascular variability: interactions and implications. Am J Physiol Regul Integr Comp Physiol, Bethesda, v. 283, n. 4, p. 815-826, Oct. 2002.

LEGRAMANTE, J. M.; et al. Hemodynamic and autonomic correlates of postexercise hypotension in patients with mild hypertension. Am J Physiol Regul Integr Comp Physiol, Bethesda, v. 282, n. 4, p. 1037-1043, Apr. 2002.

LLOYD, P. G.; YANG, H. T.; TERJUNG, R. L. Arteriogenesis and angiogenesis in rat ischemic hidlimb: role of nitric oxide. Am J Physiol Heart Circ Physiol, Bethesda, v. 28I , n. 6, p. 25282538, Dez. 2001.

LOHMEIER, T. E. Interactions between angiotensin II and baroreflexes in long-term regulation of renal sympathetic nerve activity. Circ Res, Baltimore, v. 92, n. 12, p. 1282-1284, Jun. 2003. 
MACDONALD, J. R. Potencial causes, mechanisms, and implications of post exercise hypotension. J Hum Hypertens, London, v. I6, n. 4, p. 225-236, Apr. 2002.

MAEDA, S. et al. Effects of exercise training of 8 weeks and detraining on plasma levels of endothelium-derived factors, endothelin-I and nitric oxide, in healthy young humans. Life Sci, Amsterdam, v. 69, n. 9, p. 1005-1016, Jul. 2001.

MAEDA, S. et al. Aerobic exercise training reduces plasma endothelin- I concentration in older man. J Appl Physiol, Bethesda, v. 95, n. I, p. 336-34I, Feb. 2003.

MAIORANA, A. et al. Exercise and the nitric oxide vasodilator system. Sports Med, Auckland, v. 33, n. 14, p. 1013-1035, Jan. 2003.

MANCIA, G. et al. Sympathetic activation in the pathogenesis of hypertension and progression of organ damage. Hypertension, Dallas, v. 34, n. 4, p. 724-728, Oct. 1999.

MANFREDINI, F. et al. Sport Therapy for Hypertension: Why, How, and How Much? Angiology, New York, v. 60, n. 2, p. 207-2 I6, Apr/May. 2009.

MCALLISTER, R. M. Adaptations in control of blood flow with training: splanchnic and renal blood flows. Med Sci Sports Exerc, Madison, v. 30, n. 3, p. 375-381, Marc. 1998.

MEREDITH, I. T. et al. Exercise training lowers resting renal but not cardiac sympathetic activity in humans. Hypertension, Dallas, v. 18, n. 5, p. 575-582, Nov. 1991.

MIYAI, N. et al. Antihypertensive effects of aerobic exercise in middle-aged normotensive men with exaggerated blood pressure response to exercise. Hypertension, Dallas, v. 25, n. 4, p. 507-5 |4, Jul. 2002.

MONAHAN, K. D. et al. Age-associated changes in cardiovagal baroreflex are related to central arterial compliance. Am J Physiol Heart Circ Physiol, Bethesda, v. 28I, n. I, p. 284289, Jul. 2001.

MONAHAN, K. D. et al. Central arterial compliance is associated with age- and habitual exercise- related differences in cardiovagal baroreflex sensivity. Circulation, Dallas, v. I04, n. 14, p. 1627-1632, Oct. 2001.

NEGRÃO, C. E.; RONDON, M. U. P. B. Exercício físico, hipertensão e controle barorreflexo da pressão arterial. Rev Bras Hipertens, Ribeirão Preto, v. 8, n. I, p. 89-95, Jan./Mar. 200 I.

OLIVEIRA, E. M.; ALVES, G. B.; BARAUNA, V. G. Sistema renina-angiotensina: interação geneexercício. Rev. Bras. Hipertens, Ribeirão Preto, v. 10, n. 2, p. 125-129, Abr./Jun. 2003.

O'SULLIVAN, S. E.; BELL, C. The effects of exercise and training on human cardiovascular reflex control. J Auton Nerv Syst, Amsterdam, v. 81, n. I-3, p. I6-24, Jul. 2000. 
PONTES, F. L. JR. et al. Kallikrein kinin system activation in post-exercise hypotension in water running of hypertensive volunteers. Int Immunopharmacol, Amsterdam, v. 8, n. 2, p. 26I-266, Fev. 2008.

PORTER, M. M. et al. Capillary supply of the tibialis anterior muscle in young, healthy, and moderately active men and women. J Appl Physiol, Bethesda , v. 92, n. 4, p. |45 I1457, Apr. 2002.

PRIOR, B. M. et al. Exercise-induced vascular remodeling. Exerc Sport Sci Rev, New York, v. 3I, n. I, p. 26-33, Jan. 2003.

PUDDU, P. et al. Endothelial dysfunction in hypertension. Acta cardiol, Leuven, v. 55, n. 4, p. 221-232, Aug. 2000.

RAO, S. P.; COLLINS, H. L.; DICARLO, S. E. Postexercise --adrenergic receptor hyporesponsiveness in hypertensive rats is due to nitric oxide. Am J Physiol Regul Integr Comp Physiol, Bethesda, v. 282, n. 4, p. 960-968, Apr. 2002.

RHEAUMÉ, $C$. et al. Effects of intense and prolonged exercise in insulin sensivity and glycogen metabolism in hypertensive subjects. Circulation, Dallas, v. 108, n. 2 I, p. 26532659, Nov. 2003.

RONDON, M. U. P. B. et al. Postexercise blood pressure reduction in elderly hypertensive patients. J Am Coll Cardiol, New York, v. 39, n. 4, p. 676-682, Fev. 2002.

RONDON, M. U. P. B.; BRUM, P. C. Exercício físico como tratamento não-farmacológico da hipertensão arterial. Rev Bras Hipertens, Ribeirão Preto, v. 10, n. 2, p. 134-139, Abr./ Jun. 2003.

SENITKO, A.; CHARKOUDIAN, N.; HALLIWILL, J. R. Influence of endurance exercise training status and gender on postexercise hypotension. J Appl Physiol, Bethesda, v. 92, n. 6, p. 2368-2374, Jun. 2002.

SHARMA, J. N. Does the kinin system mediate in cardiovascular abnormalities? An overview. J Clin Pharmacol, Thousand Oaks, v. 43, n. II , p. I 187-I I 95, Nov. 2003.

SHARMA, J. N.; SHARMA, J. Cardiovascular properties of the kallikrein-kinin system. Curr Med Res Opin, London, v. 18, n. I, p. 10-17, Jan. 2002.

SCHMAIER, A. H. The kallikrein-kinin system and the renin-angiotensin systems have a multilayered interaction. Am J Physiol Regul Integr Comp Physiol, Bethesda, v. 285, n. I, p. I- I3, Jul. 2003.

TADDEI, S. et al. Physical activity prevents age-related impairment in nitric oxide availability in elderly athletes. Circulation, Dallas, v. I0 I , n. 25, p. 2896-290 I, Jun. 2000. 
TAGUCHI, T. et al. Involvement of bradykinin in acute exercise-induced increase of glucose uptake and GLUT-4 translocation in skeletal muscle: studies in normal and diabetic humans and rats. Metabolism, Philadelphia, v. 49, n. 7, p. 920-930, Jul. 2000.

URATA, H. et al. Antihypertensive and volume-depleting effects of mild exercise on essential hypertension. Hypertension, Dallas, v. 9, n. 3, p. 245-252, Marc. 1987.

VAPAATALO, H.; MERVAALA, E. Clinically important factors influencing endothelial function. Med Sci Monit, Old Westbury, v. 7, n. 5, p. 1075-1085, Sep./Oct. 2001.

WHELTON, S. P. et al. Effect of aerobic exercise on blood pressure: a meta-analysis of randomized, controlled trials. Ann Intern Med, Philadelphia, v. 136, n. 7, p. 493-503, Apr. 2002.

Recebido: 13 abr. 2010 Aprovado: 24 ago. 2010

Endereço para correspondência:

Prof. Dr. Francisco Luciano Pontes Júnior Rua Arlindo Béttio, 1000 - Bairro: Ermelino Matarazzo São Paulo-SP CEP: 03828-000 